\title{
Internally Constrained Mixtures of Elastic Continua
}

\author{
STEPHEN M. KLISCH \\ Department of Mechanical Engineering, University of California at Berkeley, Berkeley, CA, USA
}

\begin{abstract}
A treatment of internally constrained mixtures of elastic continua at a common temperature is developed. Internal constraints involving the deformation gradient tensors and the common mixture temperature are represented by a constraint manifold, and an internally constrained mixture of elastic continua is associated with each unique equivalence class of unconstrained mixtures. The example of intrinsic incompressibility of each constituent first proposed by Mills is discussed.
\end{abstract}

\section{INTRODUCTION}

An internal constraint is usually regarded as a restriction on the possible motions that a material may experience. In this paper, an internal constraint for a mixture of elastic continua at a common temperature is defined as a restriction on the possible processes that the mixture constituents may experience. The general form of the internal constraint considered here, which involves both of the deformation gradient tensors, is motivated by the constraint of intrinsic incompressibility of each constituent in a saturated mixture introduced by Mills [1]. In particular, this constraint simultaneously restricts the possible values of the constituent densities. The partial time derivative of this incompressibility condition was simplified by Mills using the continuity equations and introduced into a mixture entropy inequality with a Lagrange multiplier to derive restrictions on the constitutive equations. Several authors [2-6] have studied the special constraint of intrinsic incompressibility using the approach of Mills. However, there does not exist a systematic procedure in mixture theory for introducing more general internal constraints, such as inextensibility or temperature-dependent compressibility.

The first general theory of thermomechanical constraints was developed by Green, Naghdi, and Trapp [7]. Recently, Casey [8] developed a new approach to internally constrained elastic materials, which has been extended to include thermoelastic materials by Casey and Krishnaswamy [9]. In [9], an internal constraint is represented as a constraint manifold in strain-temperature space and is used to define an equivalence relation on the set of unconstrained materials. In addition, the authors provided a prescription for the entropy, an approach that was advocated by Rivlin [10-11] and Day [12]. In a more recent paper, Krishnaswamy and Batra [13] developed prescriptions for the partial entropies of a mixture of an elastic solid and a viscous fluid at a common temperature. 
In the present paper, the method of [9] is extended to internally constrained mixtures of elastic continua at a common temperature. The basic equations for mixtures are given in Section 2, and constitutive equations for an unconstrained mixture of elastic continua at a common temperature are derived in Section 3 where the method of [13] is used to obtain prescriptions for the partial entropies. An equivalence class associated with an internal constraint for a mixture of elastic continua is defined in Section 4, which leads to a definition of a constrained mixture of elastic continua. The internal constraint of intrinsic incompressibility is discussed in Section 5.

\section{PRELIMINARIES}

Consider a mixture $C$ of two elastic constituents $C^{\alpha}$, with the superscript $\alpha=1,2$ being used to designate each constituent (the summation convention is not used on $\alpha$ ). A mixture of elastic continua naturally includes the case of a mixture of an elastic solid and an inviscid fluid, as an inviscid fluid may be regarded as an elastic material for which the free energy function only depends on the deformation gradient tensor through its determinant. A material particle of $C^{\alpha}$ occupies a position $\mathbf{X}^{\alpha}$ in a fixed reference configuration $\kappa_{0}$ and a position $\mathbf{x}^{\alpha}$ in a present configuration $\kappa$. It is assumed that there exists one particle of each $C^{\alpha}$ at each point $\mathbf{x}$ in the mixture such that $\mathbf{x}=\mathbf{x}^{1}=\mathbf{x}^{2}$. The motion of $C^{\alpha}$ and the common temperature of the mixture are defined by sufficiently smooth mappings

$$
\mathbf{x}^{\alpha}=\chi^{\alpha}\left(\mathbf{X}^{\alpha}, t\right), \quad \theta=\Theta\left(\mathbf{X}^{\alpha}, t\right),
$$

respectively, where $\theta(>0)$ is the absolute temperature of the mixture. The pair $\left\{\chi^{\alpha}, \Theta\right\}$ is referred to as a process for $C^{\alpha}$.

The density $\rho^{\alpha}$ of $C^{\alpha}$ is considered to be the average mass density of $C^{\alpha}$ over a small mixture volume. The density of the mixture is defined as

$$
\rho=\rho^{1}+\rho^{2} .
$$

The velocity of $C^{\alpha}$ is

$$
\mathbf{v}^{\alpha}=\frac{d^{\alpha}}{d t} \chi^{\alpha}\left(\mathbf{X}^{\alpha}, t\right)
$$

where the material time derivative $d^{\alpha}(\cdot) / d t$ following the motion of $C^{\alpha}$ is given for scalar or vector functions $\zeta(\mathbf{x}, t)$ and $\mathbf{w}(\mathbf{x}, t)$ by

$$
\frac{d^{\alpha} \zeta}{d t}=\frac{\partial \zeta}{\partial t}+(\operatorname{grad} \zeta) \cdot \mathbf{v}^{\alpha}, \quad \frac{d^{\alpha} \mathbf{w}}{d t}=\frac{\partial \mathbf{w}}{\partial t}+(\operatorname{grad} \mathbf{w}) \mathbf{v}^{\alpha} .
$$

The material time derivative $d(\cdot) / d t$ following the mean mixture motion is defined as

$$
\frac{d \zeta}{d t}=\frac{\partial \zeta}{\partial t}+(\operatorname{grad} \zeta) \cdot \mathbf{v}, \quad \frac{d \mathbf{w}}{d t}=\frac{\partial \mathbf{w}}{\partial t}+(\operatorname{grad} \mathbf{w}) \mathbf{v}
$$


where the mean, or barycentric, velocity $\mathbf{v}$ is defined by

$$
\rho \mathbf{v}=\rho^{1} \mathbf{v}^{1}+\rho^{2} \mathbf{v}^{2}
$$

A diffusion velocity $\mathbf{u}^{\alpha}$ and a relative velocity $\mathbf{a}$ are defined as

$$
\mathbf{u}^{\alpha}=\mathbf{v}^{\alpha}-\mathbf{v}, \quad \mathbf{a}=\mathbf{v}^{1}-\mathbf{v}^{2}
$$

From (2.4-2.5) and (2.7), we obtain the useful relationships

$$
\begin{aligned}
& \frac{d^{\alpha} \zeta}{d t}=\frac{d^{\beta} \zeta}{d t}+(\operatorname{grad} \zeta) \cdot\left(\mathbf{v}^{\alpha}-\mathbf{v}^{\beta}\right)=\frac{d \zeta}{d t}+(\operatorname{grad} \zeta) \cdot \mathbf{u}^{\alpha} \\
& \frac{d^{\alpha} \mathbf{w}}{d t}=\frac{d^{\beta} \mathbf{w}}{d t}+(\operatorname{grad} \mathbf{w})\left(\mathbf{v}^{\alpha}-\mathbf{v}^{\beta}\right)=\frac{d \mathbf{w}}{d t}+(\operatorname{grad} \mathbf{w}) \mathbf{u}^{\alpha}
\end{aligned}
$$

For each $C^{\alpha}$, the deformation gradient tensor is

$$
\mathbf{F}^{\alpha}=\partial \chi^{\alpha}\left(\mathbf{X}^{\alpha}, t\right) / \partial \mathbf{X}^{\alpha}
$$

where

$$
\mathrm{J}^{\alpha}=\operatorname{det} \mathbf{F}^{\alpha}>0
$$

The velocity gradient tensor and the rate of deformation tensor are

$$
\mathbf{L}^{\alpha}=\partial \mathbf{v}^{\alpha} / \partial \mathbf{x}, \quad \mathbf{D}^{\alpha}=\frac{1}{2}\left(\mathbf{L}^{\alpha}+\mathbf{L}^{\alpha T}\right)
$$

respectively, where the superscript $T$ denotes the transpose operator.

Assuming that internal mass exchange among the constituents is zero, the spatial forms of the balance of mass, linear momentum, angular momentum, and energy equations for $C^{\alpha}$ take the form [14]

$$
\begin{gathered}
\frac{d^{\alpha} \rho^{\alpha}}{d t}+\rho^{\alpha} \operatorname{div} \mathbf{v}^{\alpha}=0 \\
\rho^{\alpha} \frac{d^{\alpha} \mathbf{v}^{\alpha}}{d t}=\operatorname{div} \mathbf{T}^{\alpha}+\pi^{\alpha}+\rho^{\alpha} \mathbf{b}^{\alpha} \\
\mathbf{T}^{\alpha}-\mathbf{T}^{\alpha T}=\lambda^{\alpha} \\
\rho^{\alpha} \frac{d^{\alpha} \varepsilon^{\alpha}}{d t}=\rho^{\alpha} r^{\alpha}-\operatorname{div} \mathbf{q}^{\alpha}+\beta^{\alpha}+\mathbf{T}^{\alpha} \cdot \mathbf{D}^{\alpha},
\end{gathered}
$$

where $\mathbf{T}^{\alpha}$ is the partial Cauchy stress tensor, $\boldsymbol{\pi}^{\alpha}$ is the diffusive force, $\mathbf{b}^{\alpha}$ is the partial external body force, $\lambda^{\alpha}$ is the internal body couple, $\varepsilon^{\alpha}$ is the partial internal energy, $r^{\alpha}$ is the partial external heat supply, $\mathbf{q}^{\alpha}$ is the partial heat flux, and $\beta^{\alpha}$ is the internal energy supply. 
The balance of momentum for the mixture requires that

$$
\pi^{1}+\pi^{2}=0
$$

The balance of angular momentum for the mixture requires that

$$
\lambda^{1}+\lambda^{2}=0
$$

so that the total stress in the mixture is symmetric, that is,

$$
\mathbf{T}=\mathbf{T}^{1}+\mathbf{T}^{2}=\mathbf{T}^{T}
$$

When writing the balance of energy for the mixture, partial Helmholtz free-energy functions $\psi^{\alpha}$ are introduced as $\psi^{\alpha}=\varepsilon^{\alpha}-\eta^{\alpha} \theta$, where $\eta^{\alpha}$ is the partial entropy, for which a prescription will be given in Section 3. The mixture quantities $\psi, \varepsilon, r$, and $\eta$ are defined as

$$
\rho \psi=\sum_{\alpha=1}^{2} \rho^{\alpha} \psi^{\alpha}, \quad \rho \varepsilon=\sum_{\alpha=1}^{2} \rho^{\alpha} \varepsilon^{\alpha}, \quad \rho r=\sum_{\alpha=1}^{2} \rho^{\alpha} r^{\alpha}, \quad \rho \eta=\sum_{\alpha=1}^{2} \rho^{\alpha} \eta^{\alpha} .
$$

Without loss of generality, the partial stresses and diffusive forces can be written as

$$
\mathbf{T}^{\alpha}=\phi^{\alpha} \mathbf{I}+\overline{\mathbf{T}}^{\alpha}, \quad \boldsymbol{\pi}^{\alpha}=-\operatorname{grad} \phi^{\alpha}+\overline{\boldsymbol{\pi}}^{\alpha},
$$

where

$$
\phi^{\alpha}=\rho^{\alpha}\left(\psi-\psi^{\alpha}\right), \quad \phi^{1}+\phi^{2}=0
$$

and the parts of the partial stresses and diffusive forces in which $\phi^{\alpha}$ appears do not contribute to the balance equations. ${ }^{1}$ With these definitions, the balance of energy for the mixture can be expressed as [16]

$$
\rho\left(\frac{d \psi}{d t}+\eta \frac{d \theta}{d t}+\theta \frac{d \eta}{d t}\right)-\rho r+\operatorname{div} \mathbf{q}^{*}+\sum_{\alpha=1}^{2}\left(\overline{\boldsymbol{\pi}}^{\alpha} \cdot \mathbf{v}^{\alpha}-\overline{\mathbf{T}}^{\alpha} \cdot \mathbf{L}^{\alpha}\right)=0
$$

where

$$
\mathbf{q}^{*}=\mathbf{q}+\sum_{\alpha=1}^{2} \rho^{\alpha} \eta^{\alpha} \theta \mathbf{u}^{\alpha}, \quad \mathbf{q}=\mathbf{q}^{1}+\mathbf{q}^{2} .
$$

Using (2.8) and (2.12), we may, for a scalar function defined for both $C^{\alpha}$ and $C$ as in $(2.19)_{4}$, derive the useful relationship 


$$
\rho \frac{d \eta}{d t}=\sum_{\alpha=1}^{2}\left\{\rho^{\alpha} \frac{d^{\alpha} \eta^{\alpha}}{d t}-\operatorname{div}\left(\rho^{\alpha} \mathbf{u}^{\alpha} \eta^{\alpha}\right)\right\}
$$

A superposed rigid-body motion of the mixture is defined by

$$
\chi^{\alpha^{+}}\left(\mathbf{X}^{\alpha}, t^{+}\right)=\mathbf{Q}(t) \chi^{\alpha}\left(\mathbf{X}^{\alpha}, t\right)+\mathbf{c}(t), \quad t^{+}=t+c,
$$

where $\mathbf{Q}(t)$ is a proper-orthogonal second-order tensor, $\mathbf{c}(t)$ is a vector, and $c$ is a constant. The quantities $(\mathbf{Q}(t), \mathbf{c}(t), c)$ for each $C^{\alpha}$ are equal. Under a superposed rigid-body motion of an unconstrained mixture at fixed temperature, it can be shown that the following kinematic quantities transform as

$$
\mathbf{F}^{\alpha^{+}}=\mathbf{Q} \mathbf{F}^{\alpha}, \quad \mathrm{J}^{\alpha^{+}}=\mathrm{J}^{\alpha}, \quad \mathbf{D}^{\alpha^{+}}=\mathbf{Q D}^{\alpha} \mathbf{Q}^{\mathrm{T}}, \quad \rho^{\alpha^{+}}=\rho^{\alpha}, \quad \mathbf{a}^{+}=\mathbf{a}
$$

while it is assumed that

$$
\mathbf{T}^{\alpha^{+}}=\mathbf{Q} \mathbf{T}^{\alpha} \mathbf{Q}^{\mathbf{T}}, \quad \boldsymbol{\pi}^{\alpha^{+}}=\mathbf{Q} \boldsymbol{\pi}^{\alpha}, \quad \mathbf{q}^{\alpha^{+}}=\mathbf{Q q}^{\alpha}, \quad \beta^{\alpha^{+}}=\beta^{\alpha}, \quad \varepsilon^{\alpha^{+}}=\varepsilon^{\alpha}
$$

From (2.15) and (2.26-2.27), it can be concluded that

$$
r^{\alpha^{+}}=r^{\alpha}
$$

\section{ENTROPY AND RESTRICTIONS ON CONSTITUTIVE EQUATIONS}

The approach taken in this paper to develop prescriptions for the partial entropies is based on the work of Krishnaswamy and Batra [13]. In particular, these authors applied an approach advocated for materials with memory [10-11] and thermoelastic materials [9] to a mixture of an elastic solid and a viscous fluid. In this approach, a special process is defined and Part I of the Second Law of Thermodynamics is invoked to obtain the entropy prescriptions. Then, restrictions on the constitutive equations are derived from the mixture energy equation, and further restrictions are derived from invoking the Clausius-Duhem inequality as a statement of Part II of the Second Law of Thermodynamics.

We adopt the notation

$$
\Delta=\left\{\mathbf{F}^{1}, \mathbf{F}^{2}, \mathbf{G}^{1}, \mathbf{G}^{2}, \theta\right\}
$$

where $\mathbf{G}^{\alpha}=\operatorname{GradF}^{\alpha} ; \boldsymbol{\Delta}$ can be considered to be a point in a 73-dimensional Euclidean space $\mathcal{R}^{73}$. Without loss of generality, it is assumed that

$$
\begin{gathered}
\varepsilon^{\alpha}=\hat{\varepsilon}^{\alpha}(\Delta, \mathbf{a})={ }_{o} \hat{\varepsilon}^{\alpha}(\Delta)+{ }_{e} \hat{\varepsilon}^{\alpha}(\Delta, \mathbf{a}) \\
\mathbf{T}^{\alpha}=\hat{\mathbf{T}}^{\alpha}(\Delta, \mathbf{a})={ }_{o} \hat{\mathbf{T}}^{\alpha}(\Delta)+_{e} \hat{\mathbf{T}}^{\alpha}(\Delta, \mathbf{a})
\end{gathered}
$$


where

$$
\begin{array}{cc}
{ }_{o} \hat{\varepsilon}^{\alpha}(\Delta)=\hat{\varepsilon}^{\alpha}(\Delta, 0), & e^{\hat{\varepsilon}^{\alpha}}(\Delta, 0)=0 \\
{ }_{o} \hat{\mathbf{T}}^{\alpha}(\Delta) & =\hat{\mathbf{T}}^{\alpha}(\Delta, \mathbf{0}), \quad e^{\hat{\mathbf{T}}^{\alpha}}(\Delta, \mathbf{0})=\mathbf{0} .
\end{array}
$$

Furthermore, it is assumed that

$$
\mathbf{q}^{\alpha}=\hat{\mathbf{q}}^{\alpha}(\Delta, \mathbf{g}), \quad \hat{\mathbf{q}}^{\alpha}(\boldsymbol{\Delta}, \mathbf{0})=\mathbf{0},
$$

where $\mathbf{g}=\operatorname{grad} \theta$. We require that ${ }_{o} \hat{\varepsilon}^{\alpha}\left(\mathbf{I}, \mathbf{I}, \mathbf{0}, \mathbf{0}, \theta_{0}\right)=0$, where $\theta_{0}$ is the mixture temperature in the reference configuration $\kappa_{0}$. A path $P$ in the space $\mathcal{R}^{73}$ is parameterized by a real-valued function $\delta(t)$ as

$$
P(\delta)=\left\{\mathbf{F}^{1}(\delta), \mathbf{F}^{2}(\delta), \mathbf{G}^{1}(\delta), \mathbf{G}^{2}(\delta), \theta(\delta)\right\},
$$

where $\delta_{1} \leq \delta \leq \delta_{2}$. A homothermal quasi-static process is defined to be the limit of homothermal processes: ${ }^{2}$

$$
\mathbf{g}=0, \quad \dot{\delta}=\frac{d \delta}{d t}>0, \quad \dot{\delta} \rightarrow 0
$$

By defining

$$
\mathbf{v}_{*}^{\alpha}=\frac{d^{\alpha}}{d \delta} \chi^{\alpha}\left(\mathbf{X}^{\alpha}, t\right)
$$

and using $\mathbf{v}_{*}^{\alpha}$ in place of $\mathbf{v}^{\alpha}$ in (2.6-2.7) and (2.11), we obtain definitions for the quantities $\mathbf{v}_{*}, \mathbf{u}_{*}^{\alpha}, \mathbf{a}_{*}, \mathbf{L}_{*}^{\alpha}$, and $\mathbf{D}_{*}^{\alpha}$, so that

$$
\begin{aligned}
& \mathbf{v}^{\alpha}=\mathbf{v}_{*}^{\alpha} \dot{\delta}, \quad \mathbf{v}=\mathbf{v}_{*} \dot{\delta}, \quad \mathbf{u}^{\alpha}=\mathbf{u}_{*}^{\alpha} \dot{\delta}, \quad \mathbf{a}=\mathbf{a}_{*} \dot{\delta} \\
& \mathbf{L}^{\alpha}=\mathbf{L}_{*}^{\alpha} \dot{\delta}, \quad \mathbf{D}^{\alpha}=\mathbf{D}_{*}^{\alpha} \dot{\delta}
\end{aligned}
$$

Therefore, in a homothermal quasi-static process $\mathbf{v}^{\alpha} \rightarrow \mathbf{0}, \mathbf{v} \rightarrow \mathbf{0}, \mathbf{u}^{\alpha} \rightarrow \mathbf{0}, \mathbf{a} \rightarrow \mathbf{0}, \mathbf{L}^{\alpha} \rightarrow \mathbf{0}$, and $\mathbf{D}^{\alpha} \rightarrow \mathbf{0}$. Finally, in a homothermal quasi-static process, it is assumed that ${ }^{3}$

$$
\beta^{\alpha} \rightarrow 0, \quad r^{\alpha} \rightarrow 0
$$

and

$$
\beta^{\alpha} / \dot{\delta} \rightarrow 0, \quad r^{\alpha} / \dot{\delta} \rightarrow r_{\mathrm{lim}}^{\alpha}
$$


where $r_{\text {lim }}^{\alpha}$ remains finite. Hence, the energy equation (2.15) for $C^{\alpha}$ can be written in rateindependent form for a homothermal quasi-static process as

$$
\rho^{\alpha} \frac{d^{\alpha} \hat{\varepsilon}^{\alpha}(\boldsymbol{\Delta}, \mathbf{0})}{d \delta}=\rho^{\alpha} r_{\mathrm{lim}}^{\alpha}+\hat{\mathbf{T}}^{\alpha}(\boldsymbol{\Delta}, \mathbf{0}) \cdot \mathbf{D}_{*}^{\alpha},
$$

or, using (3.4-3.5), as

$$
\frac{\rho^{\alpha} r_{\lim }^{\alpha}}{\theta}=\frac{1}{\theta}\left(\rho^{\alpha} \frac{d^{\alpha}{ }_{o} \hat{\varepsilon}^{\alpha}}{d \delta}-{ }_{o} \hat{\mathbf{T}}^{\alpha} \cdot \mathbf{D}_{*}^{\alpha}\right) .
$$

Now, Part I of the Second Law of Thermodynamics is invoked to assert that the Clausius integral given by

$$
I^{\alpha}=\int_{\delta_{1}}^{\delta_{2}} \frac{\rho^{\alpha} r_{\lim }^{\alpha}}{\theta} d \delta=\int_{\delta_{1}}^{\delta_{2}} \frac{1}{\theta}\left(\rho^{\alpha} \frac{d^{\alpha}{ }_{o} \hat{\varepsilon}^{\alpha}}{d \delta}-{ }_{o} \hat{\mathbf{T}}^{\alpha} \cdot \mathbf{D}_{*}^{\alpha}\right) d \delta
$$

is path-independent. This furnishes a partial entropy function $\eta^{\alpha}=\hat{\eta}^{\alpha}(\Delta)$ such that for all homothermal quasi-static processes,

$$
\frac{d^{\alpha} \eta^{\alpha}}{d \delta}=\frac{r_{\lim }^{\alpha}}{\theta}
$$

It is assumed that the partial entropy depends only on the quantities $\Delta$ for all processes. The arbitrary constant of integration in the partial entropy is fixed by requiring $\eta^{\alpha}\left(\mathbf{I}, \mathbf{I}, \mathbf{0}, \mathbf{0}, \theta_{0}\right)=0$.

To develop restrictions on the constitutive equations, first consider the mixture energy equation (2.22) written as follows for a homothermal quasi-static process:

$$
\begin{aligned}
& \rho\left(\frac{d \psi}{d \delta}+\eta \frac{d \theta}{d \delta}+\theta \frac{d \eta}{d \delta}\right)-\rho \frac{r}{\dot{\delta}}+\operatorname{div}\left(\sum_{\alpha=1}^{2} \rho^{\alpha} \eta^{\alpha} \theta \mathbf{u}_{*}^{\alpha}\right) \\
& +\sum_{\alpha=1}^{2}\left(\overline{\boldsymbol{\pi}}^{\alpha} \cdot \mathbf{v}_{*}^{\alpha}-\overline{\mathbf{T}}^{\alpha} \cdot \mathbf{L}_{*}^{\alpha}\right)=0 .
\end{aligned}
$$

Defining, as in (3.2-3.5),

$$
\begin{aligned}
\psi & =\widehat{\psi}(\boldsymbol{\Delta}, \mathbf{a})={ }_{o} \widehat{\psi}(\boldsymbol{\Delta})+_{e} \widehat{\psi}(\boldsymbol{\Delta}, \mathbf{a}) \\
\overline{\boldsymbol{\pi}}^{\alpha} & =\overline{\boldsymbol{\pi}}^{\alpha}(\boldsymbol{\Delta}, \mathbf{a})={ }_{o} \overline{\boldsymbol{\pi}}^{\alpha}(\boldsymbol{\Delta})+_{e} \overline{\boldsymbol{\pi}}^{\alpha}(\boldsymbol{\Delta}, \mathbf{a}) \\
\overline{\mathbf{T}}^{\alpha} & =\overline{\mathbf{T}}^{\alpha}(\boldsymbol{\Delta}, \mathbf{a})={ }_{o} \overline{\mathbf{T}}^{\alpha}(\boldsymbol{\Delta})+_{e} \overline{\mathbf{T}}^{\alpha}(\boldsymbol{\Delta}, \mathbf{a})
\end{aligned}
$$

and using (2.24), (3.12) 2 , and (3.16), the mixture energy equation (3.17) can be written for a homothermal quasi-static process as 


$$
\rho\left(\frac{d_{o} \widehat{\psi}}{d \delta}+\eta \frac{d \theta}{d \delta}\right)+\sum_{\alpha=1}^{2}\left({ }_{o} \bar{\pi}^{\alpha} \cdot \mathbf{v}_{*}^{\alpha}-{ }_{o} \overline{\mathbf{T}}^{\alpha} \cdot \mathbf{L}_{*}^{\alpha}\right)=0
$$

which will be referred to as the Gibbs equation for a mixture of elastic continua. Because the partial internal energies and the partial entropies vanish in the reference configuration $\kappa_{0}$, the definitions of the partial Helmholtz free energies and (2.19) lead to the result ${ }_{o} \widehat{\psi}\left(\mathbf{I}, \mathbf{I}, \mathbf{0}, \mathbf{0}, \theta_{0}\right)=0$. Letting

$$
{ }_{o} \bar{\pi}=-{ }_{o} \bar{\pi}^{1}={ }_{o} \bar{\pi}^{2}
$$

and using (3.1), (3.18), and the chain rule, (3.21) can be written as

$$
\begin{aligned}
& \rho\left(\eta+\frac{\partial_{o} \widehat{\psi}}{\partial \theta}\right) \frac{d \theta}{d \delta}-{ }_{o} \bar{\pi} \cdot \mathbf{a}_{*}-\rho \sum_{\alpha=1}^{2}\left(\frac{\partial_{o} \widehat{\psi}}{\partial \mathbf{F}^{\alpha}} \operatorname{grad} \mathbf{F}^{\alpha} \cdot \mathbf{u}_{*}^{\alpha}\right) \\
& -\sum_{\alpha=1}^{2}\left(\overline{\mathbf{T}}^{\alpha}-\rho \frac{\partial_{o} \widehat{\psi}}{\partial \mathbf{F}^{\alpha}} \mathbf{F}^{\alpha^{\mathbf{T}}}\right) \cdot \mathbf{L}_{*}^{\alpha} \\
& +\sum_{\alpha=1}^{2} \rho \frac{\partial_{o} \widehat{\psi}}{\partial \mathbf{G}^{\alpha}} \cdot\left(\frac{d^{\alpha} \mathbf{G}^{\alpha}}{d \delta}-\operatorname{grad} \mathbf{G}^{\alpha}\left[\mathbf{u}_{*}^{\alpha}\right]\right)=0,
\end{aligned}
$$

where the notation $\frac{\partial_{o} \widehat{\psi}}{\partial \mathbf{F}^{\alpha}} \operatorname{grad} \mathbf{F}^{\alpha}$ has the component form $\frac{\partial_{o} \widehat{\psi}}{\partial \mathrm{F}_{j A}^{\alpha}} \mathrm{F}_{j A, i}^{\alpha}$ and $\operatorname{grad} \mathbf{G}^{\alpha}\left[\mathbf{u}_{*}^{\alpha}\right]$ has the component form $\mathrm{G}_{i A B, j}^{\alpha} \mathrm{u}_{* j}^{\alpha}$. Using arguments that have become standard [4-6, 13-16], the following constitutive results are deduced from (3.23):

$$
\begin{gathered}
\eta=-\frac{\partial_{o} \widehat{\psi}}{\partial \theta} \\
{ }_{o} \overline{\mathbf{T}}^{\alpha}=\rho \frac{\partial_{o} \widehat{\psi}}{\partial \mathbf{F}^{\alpha}} \mathbf{F}^{\alpha^{T}} \\
{ }_{o} \bar{\pi}=-\rho^{2} \frac{\partial_{o} \widehat{\psi}}{\partial \mathbf{F}^{1}} \operatorname{grad} \mathbf{F}^{1}+\rho^{1} \frac{\partial_{o} \widehat{\psi}}{\partial \mathbf{F}^{2}} \operatorname{grad} \mathbf{F}^{2} \\
\frac{\partial_{o} \widehat{\psi}}{\partial \mathbf{G}^{\alpha}}=0 .
\end{gathered}
$$

These equations also hold for arbitrary processes because none of the variables that appear in (3.24-3.27) depends on the relative velocity $\mathbf{a}$ or on the temperature gradient $\mathbf{g}$.

To obtain further restrictions on the constitutive equations, the Clausius-Duhem inequality is invoked as a statement of Part II of the Second Law of Thermodynamics, which in spatial form is [16]

$$
\rho \frac{d \eta}{d t}-\frac{\rho r}{\theta}+\operatorname{div}\left(\frac{\mathbf{q}^{*}}{\theta}\right) \geq 0
$$


Recalling the energy equation (2.22), relations (3.2-3.6), (3.18-3.20), and the constitutive results (3.24-3.27), we obtain from the Clausius-Duhem inequality the additional restriction

$$
e^{\widehat{\psi}}(\Delta, \mathbf{a})=0
$$

and the residual inequality

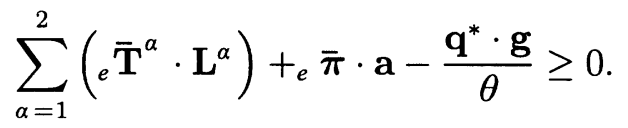

Using (3.18) and (3.29), the Helmholtz free-energy function becomes

$$
\psi=\widehat{\psi}(\boldsymbol{\Delta})=_{o} \widehat{\psi}(\boldsymbol{\Delta})
$$

\section{CONSTRAINED MIXTURES OF ELASTIC CONTINUA}

In mixture theory, Mills [1] and other authors [2-6] have studied the special constraint of intrinsic incompressibility, which simultaneously restricts the possible values of the constituent densities. For a mixture of two elastic continua, this constraint can be expressed in terms of the deformation gradient tensors of both constituents. Hence, we consider a general thermomechanical internal constraint of the form

$$
\phi\left(\mathbf{F}^{1}, \mathbf{F}^{2}, \theta\right)=0
$$

where $\phi$ is a sufficiently smooth scalar-valued function defined for the subset of the 19dimensional space $\mathcal{E}$ for which $\operatorname{det} \mathbf{F}^{\alpha}>0$ and $\theta>0$. It is assumed that the 19-dimensional vector $\left(\frac{\partial \phi}{\partial \mathbf{F}^{1}}, \frac{\partial \phi}{\partial \mathbf{F}^{2}}, \frac{\partial \phi}{\partial \theta}\right) \neq \mathbf{0}$, so that (4.1) defines a fixed 18-dimensional hypersurface $\mathcal{S} \subset \mathcal{E}$ referred to as the constraint manifold. It is assumed that $\phi$ remains invariant under superposed rigid-body motions of the mixture at fixed temperature so that (4.1) can be written in the objective forms

$$
\hat{\phi}\left(\mathbf{C}^{1}, \mathbf{F}^{1^{T}} \mathbf{F}^{2}, \theta\right)=\tilde{\phi}\left(\mathbf{C}^{1}, \mathbf{C}^{2}, \mathbf{R}^{1^{T}} \mathbf{R}^{2}, \theta\right)=0
$$

where $\mathbf{C}^{\alpha}=\mathbf{F}^{\alpha T} \mathbf{F}^{\alpha}$ and the rotation tensor $\mathbf{R}^{\alpha}$ is obtained from the polar decomposition $\mathbf{F}^{\alpha}=\mathbf{R}^{\alpha} \mathbf{U}^{\alpha}$. Although (4.2) may be more convenient for representing some types of internal constraints, in the present paper the development will proceed with the more primitive form given by (4.1).

Before proceeding, it is helpful to consider how a mixture process that satisfies (4.1) may generate different paths on $\mathcal{S}$ (see Figure 1). First, consider a material point $\mathbf{X}^{1}$ of $\mathcal{C}^{1}$. At time $t_{0}, \mathbf{X}^{1}$ occupies some spatial point $\mathbf{x}_{0}$. Also, there exists a material point $\mathbf{X}_{0}^{2}$ of $\mathcal{C}^{2}$ that occupies $\mathbf{x}_{0}$ at $t_{0}$. The vector $\left(\mathbf{F}^{1}\left(t_{0}\right), \mathbf{F}^{2}\left(t_{0}\right), \theta\left(t_{0}\right)\right)$ is defined for $\mathbf{X}^{1}$ at $t_{0}$, where $\mathbf{F}^{2}\left(t_{0}\right)$ is evaluated for $\mathbf{X}_{0}^{2}$. Clearly, the vector $\left(\mathbf{F}^{1}\left(t_{0}\right), \mathbf{F}^{2}\left(t_{0}\right), \theta\left(t_{0}\right)\right)$ identifies a point on $\mathcal{S}$. At a later time $t_{1}, \mathbf{X}^{1}$ occupies some spatial point $\mathbf{x}_{1}$; in general, $\mathbf{x}_{1} \neq \mathbf{x}_{0}$. 


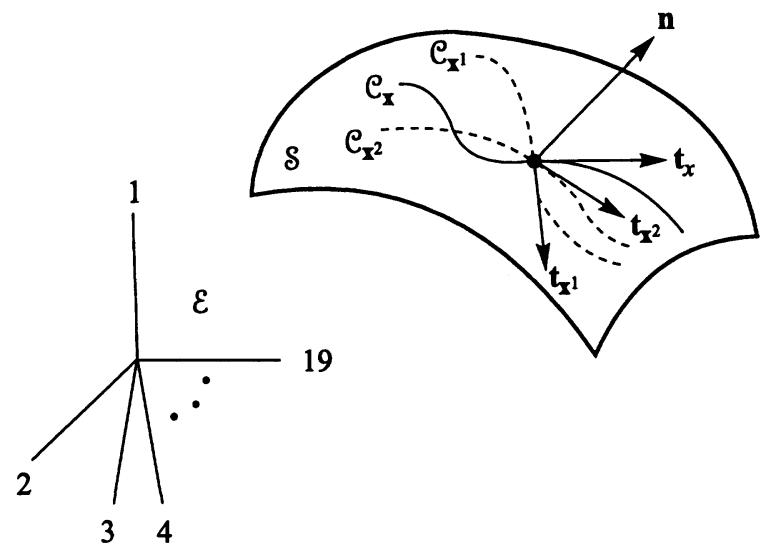

Fig. 1. The 18-dimensional constraint manifold $\mathcal{S} \subset \mathcal{E}$ defined by the internal constraint (4.1). The three curves $\mathcal{C}_{\mathbf{X}^{1}}, \mathcal{C}_{\mathbf{X}^{2}}$, and $\mathcal{C}_{\mathbf{x}}$ on $\mathcal{S}$ correspond to the three points $\mathbf{X}^{1}, \mathbf{X}^{2}$, and $\mathbf{x}$ which occupy the same spatial point at time $t$. Tangents to the curves $\mathcal{C}_{\mathbf{x}^{1}}, \mathcal{C}_{\mathbf{x}^{2}}$, and $\mathcal{C}_{\mathbf{x}}$ are $\mathbf{t}_{\mathbf{x}^{1}}, \mathbf{t}_{\mathbf{x}^{2}}$, and $\mathbf{t}_{\mathbf{x}}$, respectively, and the normal to $\mathcal{S}$ is $\mathbf{n}$.

Also, there exists a material point $\mathbf{X}_{1}^{2}$ of $\mathcal{C}^{2}$ that occupies $\mathbf{X}_{1}$ at $t_{1}$; in general, $\mathbf{X}_{1}^{2} \neq \mathbf{X}_{0}^{2}$. The vector $\left(\mathbf{F}^{1}\left(t_{1}\right), \mathbf{F}^{2}\left(t_{1}\right), \theta\left(t_{1}\right)\right)$ is defined for $\mathbf{X}^{1}$ at $t_{1}$, where $\mathbf{F}^{2}\left(t_{1}\right)$ is evaluated for $\mathbf{X}_{1}^{2}$. Once again, the vector $\left(\mathbf{F}^{1}\left(t_{1}\right), \mathbf{F}^{2}\left(t_{1}\right), \theta\left(t_{1}\right)\right)$ identifies a point on $\mathcal{S}$. Thus, the vector $\left(\mathbf{F}^{1}(t), \mathbf{F}^{2}(t), \theta(t)\right)$ defined for $\mathbf{X}^{1}$ generates a path $\mathcal{C}_{\mathbf{X}^{1}}$ on $\mathcal{S}$. A tangent vector to $\mathcal{C}_{\mathbf{X}^{1}}$ on $\mathcal{S}$ is defined using the material time derivative $d^{1}(\cdot) / d t$ following $\mathcal{C}^{1}$. In a similar fashion, the vectors $\left(\mathbf{F}^{1}(t), \mathbf{F}^{2}(t), \theta(t)\right)$ defined for $\mathbf{X}^{2}$ and $\mathbf{x}$ generate paths $\mathcal{C}_{\mathbf{X}^{2}}$ and $\mathcal{C}_{\mathbf{x}}$ on $\mathcal{S}$.

Therefore, the constraint (4.1) can be considered while one of three points are held fixed: (i) a material point $\mathbf{X}^{1}$; (ii) a material point $\mathbf{X}^{2}$; or (iii) the spatial point $\mathbf{x}$, which is simultaneously occupied by $\mathrm{X}^{1}$ and $\mathrm{X}^{2}$ at time $t$. A normal to $\mathcal{S}$ is given by

$$
\mathbf{n}=\left(\frac{\partial \phi}{\partial \mathbf{F}^{1}}, \frac{\partial \phi}{\partial \mathbf{F}^{2}}, \frac{\partial \phi}{\partial \theta}\right)
$$

for all three cases (i)-(iii). A process of the mixture that satisfies (4.1) generates three curves $\mathcal{C}_{\mathbf{X}^{1}}, \mathcal{C}_{\mathbf{X}^{2}}$, and $\mathcal{C}_{\mathbf{X}}$ on $\mathcal{S}$ corresponding to the three points $\mathbf{X}^{1}, \mathbf{X}^{2}$, and $\mathbf{x}$. Tangents to the curves $\mathcal{C}_{\mathbf{X}^{1}}, \mathcal{C}_{\mathbf{X}^{2}}$, and $\mathcal{C}_{\mathbf{x}}$ corresponding to the three cases (i)-(iii) are given by

$$
\begin{aligned}
\mathbf{t}_{\mathbf{X}^{1}} & =\left(\frac{d^{1} \mathbf{F}^{1}}{d t}, \frac{d^{1} \mathbf{F}^{2}}{d t}, \frac{d^{1} \theta}{d t}\right) \\
\mathbf{t}_{\mathbf{X}^{2}} & =\left(\frac{d^{2} \mathbf{F}^{1}}{d t}, \frac{d^{2} \mathbf{F}^{2}}{d t}, \frac{d^{2} \theta}{d t}\right) \\
\mathbf{t}_{\mathbf{x}} & =\left(\frac{\partial \mathbf{F}^{1}}{\partial t}, \frac{\partial \mathbf{F}^{2}}{\partial t}, \frac{\partial \theta}{\partial t}\right)
\end{aligned}
$$


Hence, for any process satisfying the constraint (4.1), it is necessary that

$$
\begin{aligned}
\mathbf{n} \cdot \mathbf{t}_{\mathbf{X}^{1}} & =\frac{\partial \phi}{\partial \mathbf{F}^{1}} \cdot \frac{d^{1} \mathbf{F}^{1}}{d t}+\frac{\partial \phi}{\partial \mathbf{F}^{2}} \cdot \frac{d^{1} \mathbf{F}^{2}}{d t}+\frac{\partial \phi}{\partial \theta} \frac{d^{1} \theta}{d t}=0 \\
\mathbf{n} \cdot \mathbf{t}_{\mathbf{X}^{2}} & =\frac{\partial \phi}{\partial \mathbf{F}^{1}} \cdot \frac{d^{2} \mathbf{F}^{1}}{d t}+\frac{\partial \phi}{\partial \mathbf{F}^{2}} \cdot \frac{d^{2} \mathbf{F}^{2}}{d t}+\frac{\partial \phi}{\partial \theta} \frac{d^{2} \theta}{d t}=0 \\
\mathbf{n} \cdot \mathbf{t}_{\mathbf{x}} & =\frac{\partial \phi}{\partial \mathbf{F}^{1}} \cdot \frac{\partial \mathbf{F}^{1}}{\partial t}+\frac{\partial \phi}{\partial \mathbf{F}^{2}} \cdot \frac{\partial \mathbf{F}^{2}}{\partial t}+\frac{\partial \phi}{\partial \theta} \frac{\partial \theta}{\partial t}=0
\end{aligned}
$$

for the three cases (i)-(iii). Recalling

$$
\frac{d^{\alpha} \mathbf{F}^{\alpha}}{d t}=\mathbf{L}^{\alpha} \mathbf{F}^{\alpha}
$$

$(2.4)_{2}$, and $(2.8)_{2}$, the constraint equations (4.5) can be written as

$$
\begin{aligned}
\mathbf{n} \cdot \mathbf{t}_{\mathbf{X}^{1}} & =\frac{\partial \phi}{\partial \mathbf{F}^{1}} \cdot \mathbf{L}^{1} \mathbf{F}^{1}+\frac{\partial \phi}{\partial \mathbf{F}^{2}} \cdot\left(\mathbf{L}^{2} \mathbf{F}^{2}+\operatorname{grad} \mathbf{F}^{2}[\mathbf{a}]\right)+\frac{\partial \phi}{\partial \theta} \frac{d^{1} \theta}{d t}=0 \\
\mathbf{n} \cdot \mathbf{t}_{\mathbf{X}^{2}} & =\frac{\partial \phi}{\partial \mathbf{F}^{1}} \cdot\left(\mathbf{L}^{1} \mathbf{F}^{1}-\operatorname{grad} \mathbf{F}^{1}[\mathbf{a}]\right)+\frac{\partial \phi}{\partial \mathbf{F}^{2}} \cdot \mathbf{L}^{2} \mathbf{F}^{2}+\frac{\partial \phi}{\partial \theta} \frac{d^{2} \theta}{d t}=0 \\
\mathbf{n} \cdot \mathbf{t}_{\mathbf{x}} & =\frac{\partial \phi}{\partial \mathbf{F}^{1}} \cdot\left(\mathbf{L}^{1} \mathbf{F}^{1}-\operatorname{grad} \mathbf{F}^{1}\left[\mathbf{v}^{1}\right]\right)+\frac{\partial \phi}{\partial \mathbf{F}^{2}} \cdot\left(\mathbf{L}^{2} \mathbf{F}^{2}-\operatorname{grad} \mathbf{F}^{2}\left[\mathbf{v}^{2}\right]\right) \\
& +\frac{\partial \phi}{\partial \theta} \frac{\partial \theta}{\partial t}=0
\end{aligned}
$$

where the notation $\operatorname{grad} \mathbf{F}^{\alpha}[\mathbf{a}]$ has the component form $\mathrm{F}_{i A, k}^{\alpha} a_{k}$. The middle terms of the constraint equations (4.7) can also be shown to be equal since (4.1) implies

$$
\operatorname{grad} \phi=\frac{\partial \phi}{\partial \mathbf{x}}=\frac{\partial \phi}{\partial \mathbf{F}^{1}} \operatorname{grad} \mathbf{F}^{1}+\frac{\partial \phi}{\partial \mathbf{F}^{2}} \operatorname{grad} \mathbf{F}^{2}+\frac{\partial \phi}{\partial \theta} \operatorname{grad} \theta=\mathbf{0}
$$

Although the three tangents (4.4) are, in general, not equal at a time $t$ during a given process, the relation (4.8) ensures that they all lie in the tangent space to a point on $\mathcal{S}$. The development of constrained mixtures will proceed with case (ii) (i.e., holding $\mathbf{X}^{2}$ fixed) represented by $(4.7)_{2}$. Also, it is assumed that the constraint is satisfied in the reference configuration:

$$
\phi\left(\mathbf{I}, \mathbf{I}, \theta_{0}\right)=0
$$

To define an equivalence class associated with the constraint, consider two mixtures of elastic continua, $m_{1}$ and $m_{2}$, which have common values of partial densities in the reference configuration $\kappa_{0}$. These mixtures can be considered as elements of the set $\mathcal{M}$ of all 
unconstrained mixtures, which are infinite in number. An equivalence relation associated with the constraint (4.1) is defined as

Definition 1: The mixture $m_{1}$ is equivalent to the mixture $m_{2}\left(m_{1} \sim m_{2}\right)$ if and only if

(i) $\widehat{\psi}_{m_{1}}{ }^{\alpha}(\boldsymbol{\Delta})=\widehat{\psi}_{m_{2}}{ }^{\alpha}(\boldsymbol{\Delta})\left(\Rightarrow \widehat{\psi}_{m_{1}}(\boldsymbol{\Delta})=\widehat{\psi}_{m_{2}}(\boldsymbol{\Delta})\right)$; and

(ii) $\mathbf{q}_{m_{1}}(\Delta, \mathbf{a}, \mathbf{g})=\mathbf{q}_{m_{2}}(\Delta, \mathbf{a}, \mathbf{g}), e^{\overline{\boldsymbol{\pi}}_{m_{1}}}(\Delta, \mathbf{a})={ }_{e} \overline{\boldsymbol{\pi}}_{m_{2}}(\Delta, \mathbf{a})$,

$e^{\hat{\mathbf{T}}_{m_{1}}{ }^{\alpha}}(\Delta, \mathbf{a})=e^{\hat{\mathbf{T}}_{m_{2}}{ }^{\alpha}}(\boldsymbol{\Delta}, \mathbf{a})$

for all $\left(\mathbf{F}^{1}, \mathbf{F}^{2}, \theta\right) \in \mathcal{S}$ and for all $\left(\mathbf{G}^{1}, \mathbf{G}^{2}, \mathbf{a}, \mathbf{g}\right)$.

This equivalence relation partitions the set $\mathcal{M}$ into disjoint subsets whose union is $\mathcal{M}$. The disjoint subsets are equivalence classes denoted by $M(m)=\{n \in \mathcal{M}: n \sim m\}$. Part (ii) of Definition 1 is motivated by the previous observation $[7,9]$ that an internal constraint makes no contribution to the entropy production for an internally constrained thermoelastic material.

To derive relationships among the constitutive restrictions for two equivalent mixtures $m_{1}$ and $m_{2}$, Definition 1 will be applied in two steps. Part (i) of Definition 1 will be used with the Gibbs equation (3.21) to derive relationships for the partial stresses and the diffusive forces of $m_{1}$ and $m_{2}$. Then, part (ii) of Definition 1 will be used with the Clausius-Duhem inequality (3.28) to derive a relationship for the entropy production of $m_{1}$ and $m_{2}$. First, recalling the Gibbs equation (3.21), which in light of (3.24-3.27) is satisfied for all processes, and multiplying by $\dot{\delta}$, it can be seen that

$$
\begin{aligned}
& \rho \frac{d \widehat{\psi}_{m_{1}}}{d t}=\sum_{\alpha=1}^{2}\left({ }_{o} \overline{\mathbf{T}}_{m_{1}}^{\alpha} \cdot \mathbf{L}^{\alpha}\right)+{ }_{o} \bar{\pi}_{m_{1}} \cdot\left(\mathbf{v}^{1}-\mathbf{v}^{2}\right)-\rho \eta_{m_{1}} \frac{d \theta}{d t} \\
& \rho \frac{d \widehat{\psi}_{m_{2}}}{d t}=\sum_{\alpha=1}^{2}\left({ }_{o} \overline{\mathbf{T}}_{m_{2}}^{\alpha} \cdot \mathbf{L}^{\alpha}\right)+{ }_{0} \bar{\pi}_{m_{2}} \cdot\left(\mathbf{v}^{1}-\mathbf{v}^{2}\right)-\rho \eta_{m_{2}} \frac{d \theta}{d t} .
\end{aligned}
$$

If $m_{1} \sim m_{2}$, then $\widehat{\psi}_{m_{1}}$ and $\widehat{\psi}_{m_{2}}$ match on $\mathcal{S}$ and (4.10) yields

$$
\begin{aligned}
& { }_{o} \overline{\mathbf{T}}_{m_{1}}^{1} \cdot \mathbf{L}^{1}+{ }_{o} \overline{\mathbf{T}}_{m_{1}}^{2} \cdot \mathbf{L}^{2}+{ }_{o} \overline{\boldsymbol{\pi}}_{m_{1}} \cdot \mathbf{a}-\rho \eta_{m_{1}} \frac{d \theta}{d t}={ }_{o} \overline{\mathbf{T}}_{m_{2}}^{1} \cdot \mathbf{L}^{1} \\
& +{ }_{o} \overline{\mathbf{T}}_{m_{2}}^{2} \cdot \mathbf{L}^{2}+{ }_{o} \bar{\pi}_{m_{2}} \cdot \mathbf{a}-\rho \eta_{m_{2}} \frac{d \theta}{d t}
\end{aligned}
$$

for all $\left(\mathbf{L}^{1}, \mathbf{L}^{2}, \mathbf{a}, \frac{d \theta}{d t}\right)$ satisfying $(4.7)_{2}$. Introducing a Lagrange multiplier $\lambda$ and recalling $(2.8)_{1}$ and $\operatorname{grad} \theta=0$, we obtain 


$$
\begin{aligned}
& \left({ }_{o} \overline{\mathbf{T}}_{m_{1}}^{1}-{ }_{o} \overline{\mathbf{T}}_{m_{2}}^{1}+\lambda \frac{\partial \phi}{\partial \mathbf{F}^{1}} \mathbf{F}^{\mathbf{1}^{T}}\right) \cdot \mathbf{L}^{1}+\left({ }_{o} \overline{\mathbf{T}}_{m_{1}}^{2}-{ }_{o} \overline{\mathbf{T}}_{m_{2}}^{2}+\lambda \frac{\partial \phi}{\partial \mathbf{F}^{2}} \mathbf{F}^{2^{T}}\right) \cdot \mathbf{L}^{2} \\
& +\left({ }_{o} \bar{\pi}_{m_{1}}-{ }_{o} \overline{\boldsymbol{\pi}}_{m_{2}}-\lambda \frac{\partial \phi}{\partial \mathbf{F}^{1}} \operatorname{grad} \mathbf{F}^{1}\right) \cdot \mathbf{a}-\left(\rho \eta_{m_{1}}-\rho \eta_{m_{2}}-\lambda \frac{\partial \phi}{\partial \theta}\right) \frac{d \theta}{d t}=0
\end{aligned}
$$

for all $\left(\mathbf{L}^{1}, \mathbf{L}^{2}, \mathbf{a}, \frac{d \theta}{d t}\right)$ satisfying $(4.7)_{2}$. Because the terms in parentheses of (4.12) are independent of $\left(\mathbf{L}^{1}, \mathbf{L}^{2}, \mathbf{a}, \frac{d \theta}{d t}\right)$, it is necessary and sufficient that

$$
\begin{aligned}
{ }_{o} \overline{\mathbf{T}}_{m_{2}}^{1} & ={ }_{o} \overline{\mathbf{T}}_{m_{1}}^{1}+\lambda \frac{\partial \phi}{\partial \mathbf{F}^{1}} \mathbf{F}^{1^{r}} \\
{ }_{o} \overline{\mathbf{T}}_{m_{2}}^{2} & ={ }_{o} \overline{\mathbf{T}}_{m_{1}}^{2}+\lambda \frac{\partial \phi}{\partial \mathbf{F}^{2}} \mathbf{F}^{2^{T}} \\
{ }_{o} \bar{\pi}_{m_{2}} & ={ }_{o} \bar{\pi}_{m_{1}}-\lambda \frac{\partial \phi}{\partial \mathbf{F}^{1}} \operatorname{grad} \mathbf{F}^{1} \\
\rho \eta_{m_{2}} & =\rho \eta_{m_{1}}-\lambda \frac{\partial \phi}{\partial \theta}
\end{aligned}
$$

for any two mixtures $m_{1}, m_{2} \in M(m)$ that are undergoing processes that satisfy the constraint (4.1). It should be emphasized that the relations (4.13) were obtained without invoking part (ii) of Definition 1.

Next, the Clausius-Duhem inequality (3.28) is invoked as a statement of Part II of the Second Law of Thermodynamics for $m_{2}$. Introducing a Lagrange multiplier $\lambda$ and using part (ii) of Definition 1 and (4.13), we obtain the residual entropy inequality

$$
\sum_{\alpha=1}^{2}\left({ }_{e} \overline{\mathbf{T}}_{m_{1}}^{\alpha} \cdot \mathbf{L}^{\alpha}\right)+{ }_{e} \overline{\boldsymbol{\pi}}_{m_{1}} \cdot \mathbf{a}-\frac{\mathbf{q}_{m_{1}}^{*} \cdot \mathbf{g}}{\theta}+\rho^{1}\left(\eta_{m_{1}}^{1}-\eta_{m_{2}}^{1}\right) \mathbf{a} \cdot \mathbf{g} \geq 0
$$

for any two mixtures $m_{1}, m_{2} \in M(m)$ that are undergoing processes that satisfy the constraint (4.1). Furthermore, if (4.13) is true for any two mixtures, then (4.11) is true for these two mixtures and $\widehat{\psi}_{m_{1}}$ and $\widehat{\psi}_{m_{2}}$ match on $\mathcal{S}$. However, in general, these mixtures may not be equivalent as $e_{e} \overline{\boldsymbol{\pi}}$ and ${ }_{e} \hat{\mathbf{T}}^{{ }^{\alpha}}$ may not match on $\mathcal{S}$.

Upon repeating the analysis with $(4.7)_{1}$ corresponding to holding $\mathbf{X}^{1}$ fixed, relations (4.13) are obtained except that $(4.13)_{3}$ is replaced by

$$
{ }_{o} \overline{\boldsymbol{\pi}}_{m 2}={ }_{o} \overline{\boldsymbol{\pi}}_{m 1}+\lambda \frac{\partial \phi}{\partial \mathbf{F}^{2}} \operatorname{grad} \mathbf{F}^{2}
$$

while the residual inequality (4.14) is replaced by 


$$
\sum_{\alpha=1}^{2}\left({ }_{e} \overline{\mathbf{T}}_{m_{1}}^{\alpha} \cdot \mathbf{L}^{\alpha}\right)+e \bar{\pi}_{m_{1}} \cdot \mathbf{a}-\frac{\mathbf{q}_{m_{1}}^{*} \cdot \mathbf{g}}{\theta}+\rho^{2}\left(\eta_{m_{2}}^{2}-\eta_{m_{1}}^{2}\right) \mathbf{a} \cdot \mathbf{g} \geq 0
$$

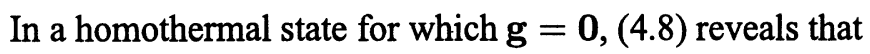

$$
\frac{\partial \phi}{\partial \mathbf{F}^{1}} \operatorname{grad} \mathbf{F}^{1}=-\frac{\partial \phi}{\partial \mathbf{F}^{2}} \operatorname{grad} \mathbf{F}^{2},
$$

so that $(4.13)_{3}$ and (4.15) are equal. Furthermore, since $\mathbf{g}=\mathbf{0 ,}(4.14)$ and (4.16) are equal. However, in a nonhomothermal state for which $\mathbf{g} \neq \mathbf{0},(4.13)_{3}$ and (4.15) are not equal and neither are (4.14) and (4.16). Thus, for arbitrary processes, (4.13-4.14) must be invoked together or, alternatively, (4.15-4.16) must be invoked together.

The above development for unconstrained mixtures of elastic continua leads to the following definition of a constrained mixture of elastic continua, which is analogous to the case of constrained thermoelastic materials [9]:

Definition 2: a constrained mixture of elastic continua $m^{\prime}$ associated with an equivalence class $M(n)$ is a mixture for which:

(i) the possible processes are those and only those that satisfy the constraint (4.1);

(ii) $m^{\prime}$ can possess the values of the quantities $\left(\psi^{\alpha}, \mathbf{q},{ }_{o} \overline{\boldsymbol{\pi}},{ }_{e} \overline{\boldsymbol{\pi}},{ }_{o} \overline{\mathbf{T}}^{\alpha},{ }_{e} \overline{\mathbf{T}}^{\alpha}, \eta\right)$ of any $m \in$ $M(n)$ when $m$ undergoes a process satisfying the constraint; and

(iii) $m^{\prime}$ can only possess values of the quantities $\left(\psi^{\alpha}, \mathbf{q},{ }_{o} \bar{\pi}, e_{e} \bar{\pi},{ }_{o} \overline{\mathbf{T}}^{\alpha}, e^{\overline{\mathbf{T}}^{\alpha}, \eta}\right)$ that are possible for any $m \in M(n)$ when $m$ undergoes a process satisfying the constraint.

From this definition, it is clear that the union of all equivalence classes associated with the constraint will generate the set of all constrained mixtures $\mathcal{M}^{\prime}$ associated with the constraint.

Consider any constrained mixture $m^{\prime} \in \mathcal{M}^{\prime}$ that is associated with an equivalence class $M(n)$. For any process that satisfies the constraint, $m^{\prime}$ possesses values of $\left(\psi_{m^{\prime}}^{\alpha}, \mathbf{q}_{m^{\prime}},{ }_{e} \bar{\pi}_{m^{\prime}},{ }_{e} \overline{\mathbf{T}}_{m^{\prime}}^{\alpha}\right)$, which are the common values of all elements in $M(n)$. Because $m^{\prime}$ can possess values of $\left({ }_{o} \bar{\pi}_{m^{\prime}},{ }_{o} \overline{\mathbf{T}}_{m^{\prime}}^{\alpha}, \eta_{m^{\prime}}\right)$ of any element in $M(n)$, from (4.13) it is evident that

$$
\begin{aligned}
{ }_{o} \overline{\mathbf{T}}_{m^{\prime}}^{1} & ={ }_{o} \overline{\mathbf{T}}_{m}^{1}+\lambda \frac{\partial \phi}{\partial \mathbf{F}^{1}} \mathbf{F}^{\mathbf{1}^{T}} \\
{ }_{o} \overline{\mathbf{T}}_{m^{\prime}}^{2} & ={ }_{o} \overline{\mathbf{T}}_{m}^{2}+\lambda \frac{\partial \phi}{\partial \mathbf{F}^{2}} \mathbf{F}^{2^{T}} \\
{ }_{o} \overline{\boldsymbol{\pi}}_{m^{\prime}} & ={ }_{o} \bar{\pi}_{m}-\lambda \frac{\partial \phi}{\partial \mathbf{F}^{1}} \operatorname{grad} \mathbf{F}^{1} \\
\rho \eta_{m^{\prime}} & =\rho \eta_{m}-\lambda \frac{\partial \phi}{\partial \theta}
\end{aligned}
$$

on $\mathcal{S}$ for any element $m \in M(n)$ undergoing the same process as $m^{\prime}$. Also, from (4.14) it is evident that 


$$
\sum_{\alpha=1}^{2}\left(e^{\overline{\mathbf{T}}_{m^{\prime}}^{\alpha}} \cdot \mathbf{L}^{\alpha}\right)+{ }_{e} \overline{\boldsymbol{\pi}}_{m^{\prime}} \cdot \mathbf{a}-\frac{\mathbf{q}_{m^{\prime}}^{*} \cdot \mathbf{g}}{\theta}+\rho^{1}\left(\eta_{m^{\prime}}^{1}-\eta_{m}^{1}\right) \mathbf{a} \cdot \mathbf{g} \geq 0
$$

on $\mathcal{S}$ for any element $m \in M(n)$ undergoing the same process as $m^{\prime}$. Upon repeating the analysis using conditions $(4.7)_{1}$ and $(4.7)_{3}$ corresponding to holding $\mathbf{X}^{1}$ and $\mathbf{x}$ fixed, respectively, we can derive the alternative and different residual inequalities

$$
\sum_{\alpha=1}^{2}\left(\overline{\mathbf{T}}_{m^{\prime}}^{\alpha} \cdot \mathbf{L}^{\alpha}\right)+{ }_{e} \bar{\pi}_{m^{\prime}} \cdot \mathbf{a}-\frac{\mathbf{q}_{m^{\prime}}^{*} \cdot \mathbf{g}}{\theta}+\rho^{2}\left(\eta_{m}^{2}-\eta_{m^{\prime}}^{2}\right) \mathbf{a} \cdot \mathbf{g} \geq 0
$$

and

$$
\sum_{\alpha=1}^{2}\left({ }_{e} \overline{\mathbf{T}}_{m^{\prime}}^{\alpha} \cdot \mathbf{L}^{\alpha}\right)+{ }_{e} \overline{\boldsymbol{\pi}}_{m^{\prime}} \cdot \mathbf{a}-\frac{\mathbf{q}_{m^{\prime}}^{*} \cdot \mathbf{g}}{\theta}+\sum_{\alpha=1}^{2} \rho^{\alpha}\left(\eta_{m^{\prime}}^{\alpha}-\eta_{m}^{\alpha}\right) \mathbf{v}^{\alpha} \cdot \mathbf{g} \geq 0
$$

However, for internal constraints that are independent of the common mixture temperature $\theta, \partial \phi / \partial \theta$ vanishes, and using $(2.19)_{4}$ and $(4.18)_{4}$ it can be shown that these residual inequalities are equal and assume the form (4.21). Furthermore, for constraints that involve only the mixture temperature $\theta$, it can also be shown that these inequalities are equal and assume the form (4.21). As a consequence of considering processes with a common mixture temperature, in the general case we cannot determine the indeterminate contributions to the partial entropies but only that of the mixture entropy as in (4.18) 4 .

Some additional results can be stated for constrained mixtures of elastic continua that are analogous to those proved for constrained thermoelastic materials since the development in the present paper parallels that of [9].

(i) By defining two constrained mixtures to be identical if the quantities $\left(\psi_{m}^{\alpha}, \mathbf{q}_{m},{ }_{e} \bar{\pi}_{m}\right.$, $\left.e^{\overline{\mathbf{T}}_{m}^{\alpha}}\right)$ are equal, then it can be seen that there exists a one-to-one relationship between equivalence classes of unconstrained mixtures and constrained mixtures.

(ii) A constrained mixture $m^{\prime}$ can be constructed from a corresponding unconstrained mixture $m$ by evaluating the quantities $\left(\psi_{m}^{\alpha}, \mathbf{q}_{m}, e \bar{\pi}_{m}, e_{e} \overline{\mathbf{T}}_{m}^{\alpha}\right)$ for $m$ on the constraint manifold $\mathcal{S}$, and noting that the quantities $\left({ }_{o} \bar{\pi}_{m^{\prime}},{ }_{o} \overline{\mathbf{T}}_{m^{\prime}}^{\alpha}, \eta_{m^{\prime}}\right)$ are specified by (4.18) where $\left({ }_{o} \bar{\pi}_{m},{ }_{o} \overline{\mathbf{T}}_{m}^{\alpha}, \eta_{m}\right)$ are evaluated on $\mathcal{S}$.

(iii) The invariance conditions for a constrained mixture $m^{\prime}$ associated with an equivalence class $M(n)$ must be modified. For any $m \in M(n)$, recalling $(2.19)_{4},(2.27)_{1},(2.27)_{2}$, (2.28), (3.16), and the fact that the entropy is defined to vanish in the reference configuration, it can be seen that under a superposed rigid-body motion at fixed temperature

$$
{ }_{o} \overline{\mathbf{T}}_{m}^{\alpha+}=\mathbf{Q}_{o} \overline{\mathbf{T}}_{m}^{\alpha} \mathbf{Q}^{T}, \quad{ }_{o} \overline{\boldsymbol{\pi}}_{m}^{+}=\mathbf{Q}_{o} \overline{\boldsymbol{\pi}}_{m}, \quad \eta_{m}^{+}=\eta_{m} .
$$

It is also assumed that $\phi$ remains invariant under a superposed rigid-body motion at fixed temperature so that 
$\left(\frac{\partial \phi}{\partial \mathbf{F}^{\alpha}}\right)^{+}=\mathbf{Q} \frac{\partial \phi}{\partial \mathbf{F}^{\alpha}}, \quad\left(\operatorname{grad} \mathbf{F}^{1}\right)^{+}=\mathbf{Q} \operatorname{grad} \mathbf{F}^{1} \mathbf{Q}^{T}, \quad\left(\frac{\partial \phi}{\partial \theta}\right)^{+}=\frac{\partial \phi}{\partial \theta}$.

However, the inherent arbitrariness of the Lagrange multiplier $\lambda$ prohibits the assumption that $\lambda$ remains properly invariant under a superposed rigid-body motion at fixed temperature. Hence, for a constrained mixture $m^{\prime},(4.18)$ and (4.22-4.23) give

$$
\begin{aligned}
& { }_{o} \overline{\mathbf{T}}_{m^{\prime}}^{\alpha+}=\mathbf{Q}\left({ }_{o} \overline{\mathbf{T}}_{m^{\prime}}^{\alpha}+\left(\lambda^{+}-\lambda\right) \frac{\partial \phi}{\partial \mathbf{F}^{\alpha}} \mathbf{F}^{\alpha^{\mathbf{T}}}\right) \mathbf{Q}^{\mathbf{T}} \\
& { }_{o} \overline{\boldsymbol{\pi}}_{m^{\prime}}^{+}=\mathbf{Q}\left({ }_{o} \overline{\boldsymbol{\pi}}_{m^{\prime}}-\left(\lambda^{+}-\lambda\right) \frac{\partial \phi}{\partial \mathbf{F}^{1}} \operatorname{grad} \mathbf{F}^{1}\right) \\
& \rho \eta_{m^{\prime}}^{+}=\rho \eta_{m^{\prime}}-\left(\lambda^{+}-\lambda\right) \frac{\partial \phi}{\partial \theta} .
\end{aligned}
$$

\section{INTRINSIC INCOMPRESSIBILITY}

A commonly used constraint in the theory of mixtures is that of intrinsic incompressibility first proposed by Mills [1] and studied by other authors [2-6]. Each constituent $\mathcal{C}^{\alpha}$ is assumed to be separable from the others with constant (true) density $\rho^{\alpha T}$ defined as the mass of $\mathcal{C}^{\alpha}$ per unit volume of $\mathcal{C}^{\alpha}$. Upon addition in forming the mixture, it is assumed that the volumes of $\mathcal{C}^{\alpha}$ add to form the volume of the mixture. With these assumptions, Mills [1] derived an equation that is a special form of the general internal constraint represented by (4.1):

$$
\frac{\rho^{1}}{\rho^{1 T}}+\frac{\rho^{2}}{\rho^{2 T}}=1
$$

Using the local form of the continuity equation, $\rho^{\alpha} J^{\alpha}=\rho_{0}^{\alpha},(5.1)$ can be written in the form (4.1) as

$$
\frac{\rho_{0}^{1}}{\rho^{1 T} \operatorname{det} \mathbf{F}^{1}}+\frac{\rho_{0}^{2}}{\rho^{2 T} \operatorname{det} \mathbf{F}^{2}}-1=0
$$

Recalling

$$
\frac{\partial \operatorname{det} \mathbf{F}^{\alpha}}{\partial \mathbf{F}^{\alpha}}=\left(\operatorname{det} \mathbf{F}^{\alpha}\right) \mathbf{F}^{\alpha-T}
$$

equations (4.18) become

$$
{ }_{o} \overline{\mathbf{T}}_{m^{\prime}}^{1}={ }_{o} \overline{\mathbf{T}}_{m}^{1}-\lambda \frac{\rho^{1}}{\rho^{1 T}} \mathbf{I}
$$




$$
\begin{aligned}
& { }_{o} \overline{\mathbf{T}}_{m^{\prime}}^{2}={ }_{o} \overline{\mathbf{T}}_{m}^{2}-\lambda \frac{\rho^{2}}{\rho^{2 T}} \mathbf{I} \\
& { }_{o} \overline{\boldsymbol{\pi}}_{m^{\prime}}={ }_{o} \bar{\pi}_{m}-\lambda \frac{\operatorname{grad} \rho^{1}}{\rho^{1 T}} .
\end{aligned}
$$

In deriving $(5.4)_{3}$, it was assumed that $\operatorname{grad} \rho_{0}^{1}=0$ is consistent with (4.8); however, this assumption is not necessary when using the approach by Mills [1]. This apparent contradiction may be resolved in one of two ways. By defining the constraint (4.1) to be of the form

$$
\phi\left(\mathbf{F}^{1}, \mathbf{F}^{2}, \rho_{0}^{1}, \rho_{0}^{2}, \theta\right)=0
$$

the procedure of Section 4 can be used with the constraint (5.2) to obtain the results (5.4) without requiring that $\operatorname{grad} \rho_{0}^{1}$ vanish. Alternatively, we may choose a reference configuration for a mixture subject to (5.4) for which the initial constituent densities are homogeneous. For this constraint, we note that the indeterminate terms in $(4.13)_{3}$ and (4.15) are equal as are the three residual inequalities (4.19-4.21) because the constraint (5.2) is independent of the common mixture temperature. Hence, the general treatment of internally constrained mixtures of elastic continua reduces to the theory of intrinsic incompressibility of each constituent as proposed by Mills [1] and used by other authors [2-6].

Acknowledgments. The author gratefully acknowledges discussions regarding the content of this paper with Professor James Casey, Department of Mechanical Engineering, U.C. Berkeley.

\section{NOTES}

1. For a discussion concerning the decompositions (2.20), see [15].

2. As noted in [13], a homothermal quasi-static process is not an actual process of the mixture, but rather a limit of actual processes.

3. The assumption $\beta^{\alpha} \rightarrow 0$ is motivated by the results of [16], which, for the mixture under consideration in the present paper, state that $\beta^{\alpha}$ vanishes when both the temperature gradient and the relative velocity are zero.

\section{REFERENCES}

[1] Mills, N.: Incompressible mixtures of Newtonian fluids. International Journal of Engineering Science, 4, 97-112 (1966).

[2] Mills, N. and Steel, T. R.: Non-linear deformations of a mixture of two isotropic elastic solids. Acta Mechanica, 9, 229-244 (1970)

[3] Craine, R. E.: Oscillations of a plate in a binary mixture of incompressible Newtonian fluids. International Journal of Engineering Science, 9, 1177-1192 (1971).

[4] Bowen, R. M.: Incompressible porous media models by use of the theory of mixtures. International Journal of Engineering Science, 18, 1129-1148 (1980).

[5] Rajagopal, K. R. and Tao, L.: Mechanics of Mixtures, World Scientific, Singapore, 1995.

[6] Atkin, R. J. and Craine, R. E.: Continuum theories of mixtures: Applications. Journal of the Institute of Mathematics and Its Applications, 17, 153-207 (1976).

[7] Green, A. E., Naghdi, P. M., and Trapp, J. A.: Thermodynamics of a continuum with internal constraints. International Journal of Engineering Science, 8, $891-908$ (1970). 
[8] Casey, J.: A treatment of internally constrained materials. Journal of Applied Mechanics, 62, 542-544 (1995).

[9] Casey, J. and Krishnaswamy, S.: A characterization of internally constrained thermoelastic materials. Mathematics and Mechanics of Solids, 3, 71-89 (1998).

[10] Rivlin, R. S.: Comments on some recent researches in thermomechanics. Recent Advances in Engineering Science, 8, 1-23 (1973).

[11] Rivlin, R. S.: Reflections of certain aspects of thermomechanics. Paper presented at Contributi del Centro Linceo Interdisiplinare di Scienze Matematiche e loro Applicazioni, No. 76- Meeting on: Finite thermoelasticity, Rome 30 May - 1 June, 11-43, (1986).

[12] Day, W. A.: The Thermodynamics of Simple Materials with Memory, Springer, Berlin/Heidelberg/New York, 1972.

[13] Krishnaswamy, S. and Batra, R. C.: A thermomechanical theory of solid-fluid mixtures. Mathematics and Mechanics of Solids, 2, 143-151 (1997).

[14] Atkin, R. J. and Craine, R. E.: Continuum theories of mixtures: Basic theory and historical development. Quarterly Journal of Mechanics and Applied Mathematics, 29, 209-244 (1976).

[15] Green, A. E. and Naghdi, P. M.: A note on mixtures. International Journal of Engineering Science, 6, 631-635 (1968).

[16] Craine, R. E., Green, A. E., and Naghdi, P. M.: A mixture of viscous elastic materials with different constituent temperatures. Quarterly Journal of Mechanics and Applied Mathematics, 23, 171-184 (1970). 